\title{
The Trend of Logic and Foundation of Mathematics in Japan in 1991 to 1996
}

\section{Yuzuru KakUdA ${ }^{*}$ and Kanji Namba** and Nobuyoshi MотонаSHI ${ }^{* * *}$}

During the term from 1991 to 1996 the alternation of generations has occurred in the logic society in Japan. The main reason of the alternation is the shift of the central concern in logic to new logical reasonings due to the advent of computer. By the request of the computer science many logicians has been aware of its applications and many interesting branches of logic have emerged as its result. Also, this trend has shed new light on existing subjects such as intermediate logic, modal logic and substructural logic. It is known by the fact that many of logic meetings held in Japan during the term had connections with information science to a greater or less extent.

Traditionally, the logicians in Japan is working in the following four divisions :

1. Non-classical logic systems

2. Proof theory

3. Recursion theory

4. Set theory

5. Model theory

In addition to the above, some logicians has contributed to the following interdisciplinary branch.

6. Non-standard analysis

7. Applications to theoretical computer science

Let us explain the trend of Logic and Foundations of Mathematics in Japan according to the above classification in due order except the last one. Though the last one should be reported in Computer Science, we would like to remark that the organization of the group SLACS, which devotes to the frontier between logic and computer science, was much of credit to Susumu Hayashi. The field successfully

* Department of Computer and Sytem Engineering, Faculty of Engineering, Kobe University, Nada, Kobe, Japan

** Graduate School of Mathematical Sciences, University of Tokyo, Komaba, Tokyo, Japan

*** Institute of Mathematics, Tsukuba University, Tsukuba, Japan 
took root in the coutry with efforts of his and other logicians and computer scientsists.

\section{Non-classical logic}

During the term the situation of research of non-classical logic may be expressed by the key words interdisciplinary and internationalization. The research of nonclassical logic itself has 25 years tradition in Japan, pioneered by Katuzi Ono in Nagoya, and mainly succeeded by the research group MLG led by Hiroakira Ono. The research of this branch had not been necessarily a central theme in the society of logicians. However, in those five or six years, by the request from the theory of programming language and verification of programming, the importance of logic has been deeply recognized. Under the circumstance the research of non-classical logic has been more and more active, and the population of researchers in the field has extended rapidly. Many researchers in the field has related to information science, and developed their work under the consciousness of applications to information science. This is the worldwide tendency, and several international meetings concerning the boundary between logic and information science was held in Japan.

Saburo Tamura studied substructural logic from a lattice structural point of view more than 20 years ago in Japan. However Yuichi Komori and Hiroakira Ono firstly studied it as a framework of a logical system itself in the country, and now Ono is studying substructural logic from the point of view that it is a framework to treat various non-classical logics unifingly. Mituhiro Okada obtained some interesting results concerning the relationship between the completeness and the cutelimination of linear logic.

M. Zakharyaschev introduced an epoch-making method in the branch of modal logic, and he obtained several important results such as decidability and finite model property. F. Wolter applied the method to tense logic and modal intuitionistic logic, and got several interesting results. Nobuyuki Suzuki and Tateya Shimura has been working in modal predicate logic. Mamoru Kaneko and Takashi Nagshima has been working in an application of logic of knowledge to the theory of game. Michio Takano obtained result about subformula property.

On the boundary between $\lambda$ calculus and logic Sachio Hirokawa got results concerning principal type scheme, and Yuuich Komori has studied weak implication logics.

\section{Proof theory}

Proof theory is the branch of logic with the oldest tradition in Japan. The Fundamental Conjecture of Gaisi Takeuti inspired many logicians in Japan to make efforts to solve it. Takeuti introduced the concept ordinal diagram to give the consistency proof of the system of Peano Arithmetic with $\prod_{1}^{1}$ comprehension axiom. 
Toshiyasu Arai is now developing an ordinal notation system to get the consistency proof along Takeuti's line. At least he got the consistency proof of $\sum_{2}^{1}$ comprehension. The work will be published in a complete book style in the near future. It seems that Arai's idea advances remarkable progress enough to give an essential solution of Takeuti's conjecture.

Other than the above, Arai made a slow growing analogue to Buchholz' proof. It can be considered as a proof theoretic interpretation of the hierarchy comparison theorem of Girard. (Arai [1]) He also gave a new method to generate functions in subrecursion theory. (Arai [2]) Ryu Hasegawa gave an ordinal notation by considering the certain class of algebras, and showed that the every ordinal up to Rahthuen-Weiremann's ordinal is represented by the order type of an algebra in the class and vice versa. By using the ordinal notation he showed that divisibility orderings are independent from subsystems of the second-order arithmetic. (Hasegawa [1]) He also considered a logical aspect of parametricity. (Hasegawa [2]) Makoto Kikuchi gave several other proofs of the second incompleteness theorem of Gödel by discussing Boolos' proof concerning Berry's paradox, Kreisel's proof and Kolmogorov complexity. (Kikuhci [1], [2], [3]) Kazuyuki Tanaka has worked actively in the field Reverse Mathematics proposed by H. Friedman and S. Simpson. (Tanaka [1], [2], [3], [4], [5], [6]) Susumu Hayashi and Mariko Yasugi discussed a functional system with transfinitely defined types and she proved the normalization theorem of the system. (Hayashi [1], Hayashi [1], Ryu [1], Nakata [1]) Noriko Arai investigated the complexity of proof of Gentzen style, focusing on the cut rule. (N. Arai [1] and [2])

\section{Recursion theory and Complex theory}

In classical recursion theory, the main concern has been classification of recursively enumerable sets. Especially, the degree structure of recursively enumerable sets has been extensively studied since Friedberg and Muchnik solved the problem raised by E. Post. In Japan, however, recusrsion theory was first studied in the context of effective descriptive set theory by people surrounding M. Kondo such as T. Tugué, H. Tanaka, Y. Suzuki, etc. Then, G. Takeuti opened the door to the generalized recursion theory with his pioneering work on the theory of recursive functions of ordinal numbers. Although these area is still traditional in Japan, many of the recent works in recursion theory are relating to the problems arising in the theory of computational complexity. Several important results have been obtained in this field (Ogiwara [1], Toda [1] and Toda [2]).

Proof theoretic approach is known to be important to attack the problems in complexity theory. By G. Takeuti and et al., various kind of systems of weak arithmetic are investigated corresponding to the complexity classes such as $\mathrm{NC}$, LSPACE (Takeuti [1]-[11]). Analyzing Ajtai's methods, G. Takeuti and M. 
Yasumoto [12] developed the theory of Boolean valued models on a nonstandard model of Peano Arithmetic. Using this theory, they showed that if there is a generic extension which is a model of $S_{2}$ then it holds that $P \neq N P$.

After the work of Baker-Gill-Solovay, most of the relativized problems of separation like $P^{A} \neq N P^{A}$ have been solved. But, it is an interesting problem to clarify the recursion theoretic complexity of the set of oracles used for separating given complexity classes. Tanaka [1]-[2] and Kudoh [1] studied such kind of problems from the standpoint of descriptive set theory. Relativization problems lead to the notion of reducibility in natural way. The polynomial time Turing reducibility as well as polynomial time many-one reducibility has been extensively studied. J. Shinoda and T. Slaman proved the first order theory of the polynomial time degrees of recursive sets is undecidable. Since then, the structures of polynomial time degrees have been investigated based on various kind of reducibilities (Aoki-Shinoda-Tsuda [1], [2] and Shinoda [1]).

Concerning the Kolmogorov complexity, Kojiro Kobayashi [2], [3] observed the differences between malignness and a riori-ness and between malign measures and universal distributions.

In classical recursion theory, the generic degrees play an important role in the global theory of the Turing degrees. Kumabe [6] is a good reference of this field. Masahiro Kumabe [1]-[5] obtained various important results on the structure of generic degrees.

In the generalized recursion theory, it was proved by J. Shinoda and T. Slaman that under the assumption of the continuum hypothesis the theory of the Kleene degrees of the sets of the reals is undecidable. Hisato Muraki [1] continues to study the structure of the Kleene degrees under the axiom of constructibility. It should be noticed that his methods are closely related to the ones developed in the computational complexity.

\section{Set theory}

Gaisi Takeuti is the first logician who studied set theory systematically in Japan. Under his influence Kanji Namba started to work in the theory of large cardinals. He obtained several interesting results on measurable cardinals and results related to forcing. Yuzuru Kakuda took over the tradition and obtained results related to ideals on sets. Under those circumstances set theoreticians in Japan has been interested in the research of ideals or filters on sets. This tendency is now more noticeable by coming back to Japan of Yo Matsubara. It should be also remarked that there is an another tendency, led by Katsuya Eda and Shizuo Kamo, which tries to apply set theoretical techniques to the theory of infinite abelian groups and general topology. (Eda [1], [2], [3] and [4]) Set theories based on non-classical logics has been investigated, set theory based on stationary 
logic by Kakuda, intuitionistic logic and modal logic by Satoko Chitani and linear logic by Masaru Shirahata (Shirahata [1]).

Saturatedness of ideals on $P_{\kappa} \lambda$ was investigated in Abe [3], Matsubara [1] and [4]. In Matsubara [1] Matsubara showed that the existence of sufficiently saturated ideal on $P_{\kappa} \lambda$ implies the singular cardinal hypothesis up to $\lambda$. He and D. Burke worked on the conjecture that the non-stationary ideal on $P_{\kappa} \lambda$ is not saturated unless $\boldsymbol{x}=\lambda=\boldsymbol{\aleph}_{1}$. (Matsubara [4]). In Matsubara [2], he and D. Burke studied the relationship between saturated (or presaturated) ideals on $P_{k} \lambda$ and some combinatorial principles. Nowhere preciptousness on ideals on $P_{k} \lambda$ was investigated by Matsubara and Masahiro Shioya. (Matsubara [3]) Yasuo Yoshinobu showed that the existence of a preciptous ideal on the successor of a singular cardinal implies the existence of an inner model with a strong cardinal. He also showed that the same kind of result is obtained by the existence of a preciptous ideal on the successor of a certain large cardinal. (Yoshinobu [1]) He also showed that the preciptousness of a stationary tower of Woodin implies an inner model with Woodin cardinals. (Yoshinobu [2]) Weak normality of filters on $P_{\kappa} \lambda$ was investigated in Abe [1], [2], Shioya [1]. Shizuo Kamo has intensively investigated properties of ideals on $\omega$ and related properties. (Kamo [1], [2]) He also investigated Mycielsky ideals and solved affirmatively the problem of Roslanowski. (Kamo[3]) Works related to $P_{k} \lambda$ other than the stated above are in Kamo [4], [5] and Shioya [3].

Yoshihumi Yuasa has investigated entangled linear orders (Yuasa [1], [2]), Tadatoshi Miyamoto has worked in the field related to proper forcing, in Miyamoto [1], he treated thepresevatuon of $\omega_{1}$-Souslin tree via forcing with countable support iteration. Cardinal invariants was studied in Kada [1] and [2]. Masazumi Hanazawa has exclusively studied properties on trees in set theory almost for the last two decades, and he got many detailed results. (Hanazawa [1], [2], [3] and [4]).

\section{Model theory}

In Japan Nobuyoshi Motohashi started to study model theory systematically, and Akito Tsuboi has had it take root in the country. Gradually young logicians are now engaged in this branch. In those five years the model theorists in Japan have mainly worked in the field related to general stable theories. The following theme

Can a property of a theory be preserved by a certain operation to the theory?

is a common concern among them. For example, H. Kikyo and Tsuboi (Kikyo [2]) and Wakai [1] investigated a preservation problem by adding an unary predicate symbol to a theory. Also, Tsuboi's investigation (Tsuboi [5]) how the union of two theories can have their original properties may be considered along this line.

Study on model theoretic structure of groups are there in Ikeda [4], Tsuboi [2], 
Tsuboi [3]. Tanka has studied $\omega$-stable groups by the group theoretic method.

Masanori Itai showed that the strong Martin conjecture holds for all seven types $\omega$-stable theory with continuum many countable models except one case. (Itai [1]) He also showed that $K$-generic projective planes have Morley rank either two or infinity. (Itai [2]) H. Kikyo obtained an interesting result concerning strongly nonmultidimmensinal theories. (Kikyo [1]) K. Ikeda obtained results about minimal models, in which he extended the result of Marcus. (Ikeda [1], Ikeda [2]) $\mathrm{He}$ and Tsuboi introduced the concept almost total elementary map as a general notion of automorphism of mathematical structures, and showed that every almost elementary map of $\aleph_{1}$-categorical structures is an automorphism. (Ikedai [3]) Ikeda, Pillay and Tsuboi showed that a theory with 3 countable models can define a linear order, provided that a certain condition is held in the theory. (Ikeda [5]) This concerns the famous conjecture that a theory with only finite countable models can define a linear order.

\section{Non-standard analysis}

It is much of credit to Masahiko Saito that non-standard analysis became popular in Japan. Now, several logicians and mathematicians has been working in this area.

H. Akiyama gave a non-standard approach to diffusions on manifolds and non-standard heat kernels, and also obtained a non-standard representation of generalized sections of vector bundles. (Akiyama [1], [2]) T. Nakamura got a non-standard representation of Feyman's path integrals (Nakamura [1]), and S. Nagamachi and T. Nishsimura got an application to Fermion Fock spaces. (Nagamachi [1]) M. Yasumoto has worked in the non-standard approach to the number theory and obtained some interesting results concerning about irreducible polynomials. (Yasumoto $[1],[2]$ ) M. Ozawa developed forcing in nonstandard analysis and tried to apply to functional analysis. (Ozawa [1], [2], Hinokuma [1]) Yuzuru Kakuda proposed a syntactical method of non-standard analysis based on consistent types (consistent sets of formulas) ten years ago. Inspired by the idea, Mariko Yasugi investigated a proof theoretic method and formalized a system based on infinitary logic in which non-standard analysis can be sufficiently developed. (Mori [1])

\section{Bibiography}

\section{Abe, Yoshihiro}

1. Weakly normal filters and large cardinals, Tsukuba J. Math. 16, 487-494, 1992

2. Weakly normal ideals on $P_{\kappa} \lambda$ amd the singular cardinal hypothesis, Fund. 
Math. 143, 97-106, 1993

3. Saturation of fundamental ideals on $P_{\kappa} \lambda$, J. Math. Soc. Japan, 48, 511-521, 1996

\section{Akiyama, Hiroshi}

1. A nonstandard approach to diffusions on manifolds and nonstandard heat kernels, in "Analysis, Probability and Mathematical Physics : Contributions of Nonstandard Analysis" (S. Albeverio, W.A.J. Luxemburg and M. Wolff, eds.), Mathematics and Its Applications Vol. 314, pp. 143-148, Kluwer Academic Publishers, Dordrecht, 1995

2. Nonstandard representations of generalized sections of vector bundles, Osaka J. Math., 32, 817-822, 1995

\section{Aoki, Kunimasa}

1. Strong minimal pair theorm for the honest polynbomial degrees of $\Delta_{2}^{0}$ low sets, Journal of Mathematical Society of Japan, Vol.44, No. 3, 505-514, 1992 (with J. Shinoda, T. Tsuda)

2. On $\mathrm{II}_{2}$ theories of $\mathrm{hp}-\mathrm{T}$ degrees of low sets, Theoretical Computer Science, Vol. 123, 315-327, 1994 (with J. Shinoda, T. Tsuda)

\section{Arai, Noriko}

1. A proper hierarchy of propositional sequent calculi, Theoretical Computer Science 159, 343-354. 1996

2. Tractability of Cut-free Gentzen Type Propositional Calculus with Permutation Inference, to appear in Theoretical Computer Science.

\section{Arai, Toshiyasu}

1. A slow growing analogue to Buchholz' proof, Annals of Pure and Applied Logic 54, 101-120, 1991

2. Variations on a theme by Weiermann, To appear in Journal of Symbalic Logic

\section{Eda, Katsuya}

1. A factor of singular homology, Tsukuba J. Math., 15, 351-387,1991 (with K. Sakai)

2. Applications of set theory to abelian groups Sugaku 43, 128-137, 1991

3. Abelian groups of continuous functions and their duals, Topology Appl. 53, 131-151, 1993 (with S. Kamo and H. Ohta)

4. Tightness about sequential fans and combinatrial properties, J. Math. Soc. Japan, to appear (with M. Kada and Y. Yuasa) 


\section{Fukuzaki, Kenji}

1. Equivalence of the induction schema and the least number principle for open formulas, submitted (with K. Aoyama)

\section{Hanazawa, Masazumi}

1. Probabilistic minimax Search of a Game Tree, Proceedings of the Faculty of Science, Tokai University, Vol. XXVII, 1-16, 1992

2. Generating most parsimonious reconstructions on a tree: A generalization of the Farris-Swofford-Maddison method, with Hiroshi Narushima and Nobuhiro Minaka, Discrete Applied !athematics 56, 245-265, 1995

3. CS-Aronszajn trees under the negation of the Continuum Hypothesis, Proceedings of the School of Science, Tokai University, Vol. XXX, 1-8, 1995

4. On a Refinement of Anti-Souslin Tree Property, Tsukuba Journal of Math ematics, Vol. 20 No. 1, June 1996, 1996

\section{Hasegawa, Ryu}

1. Well-ordering of algebras and Kruskal's theorem, in : Logic, Language and Computation, Festschrift in Honor of Satoru Takasu, N.D. Jones, M. Hagiya, M. Sato, eds., Lecture Notes in Computer Science 792, Springer, 133-172. 1994

2. A logical aspect of parametric polymorphism, in : Computer Science Logic, 9th International Workshop CSL'95, H.K. B"uning, ed., Paderborn, Germany, 1995, Lecture Notes in Computer Science 1092, Springer, 291-307, 1995

\section{Hayashi, Susumu}

1. A Functional System with Transfinitely Defined Types, Logic, Language and Computation (ed. N. Jones, M. Hagiya, M. Sato) LNCS 792 Springer Verlag, 31-60, 1994 (with M. Yasugi)

2. Interpretation of Transfinite Recursion and Parametric Abstraction in Types, Words, Languages and Combinatorics II (ed. M. Ito and H. Jurgensen) World Scientific, 452-464,1994 (with M. Yasugi)

\section{Hinokuma, T.}

1. Conversion from nonstandard matrix algebras to standard factors of type $l_{1}$. Illinois J. Math. 37-1, 1-1, 1993 (with M. Ozawa) 


\section{Ikeda, Kouichiro}

1. Minimal models of minimal theories, Tsukuba Journal of Mathematics, Vol. 17, No. 2 , 491-496, 1993

2. A remark on minimal models, Tsukuba Journal of Mathematics, Vol. 19, No. 2, 269-272, 1995

3. Almost total elementary maps, Mathematical Logic Quarterly, Vol. 41, No. 3, 353-361, 1995 (with A. Tsuboi)

4. On theories having a small Galois group, Kobe Journal of Mathematics, Vol. 13, No. 1 , 61-67, 1996

5. On theories having three countable models, submitted (with A. Tsuboi, A. Pillay)

\section{Itai, Masanori}

1. On the strong Martin conjecture, JSL Vol 56, No. 3, 862-875, 1991

2. K-generic projective planes have Morley rank two or infinity, Mathematical Logic Quarterly, vol 40, 143-152, 1994 (with T. Boldwin)

\section{Kada, Masaru}

1. Cardinal invariants about shrinkability of unbounded sets. to appear in Topology Appl (wuth Y. Yuasa)

2. New cardinal invariants related to pseudo-Dirichlet sets. to appear (with S. Kamo)

\section{Kamo, Shizuo}

1. Ideals on $\omega$ which are obtained from Hausdorf gaps, Tsukuba Journal Math. Vol. 15, No. 2, 523-528, 1991

2. Almost coinciding families and gaps in $P(w)$, J. Math. Soc. Japan, Vol. 45, No. 2, 357-368,1993

3. Some remarks about Mycielski ideals, Clloquium Math., Vol. LXV (2), 291299, 1993

4. Remarks on $P_{\kappa} A$ combinatorics, Fundamenta Math. Vol. 145, No. 1, 141151,1994

5. Ineffability and partition property on $P_{\kappa} A$, to appear in J. Math. Soc. Japan

6. See also Eda [3] and Kada [2]

\section{Kaneko, $M$.}

1. Game logic and its applications 1, II, to appear in Studia Logica (with T. Nagashima) 


\section{Kikuchi, Makoto}

1. A note on Boolos' proof of the incompleteness theorem, Mathematcal Logic Quaterly 40, No. 4, 528-532, 1994

2. On formalization of model-theoretic proofs of Gödel's theorems, Notre Dame Journal of Formal Logic 35, No. 3, 403-412, 1994, with K. Tanaka

3. Kolmogorov complexity and the second incompleteness theorem, to appear in Archive for Mathematical Logic

\section{Kikyo, Hirotaka}

1. Strongly nonmultidimensional theories, J. Math. Soc. Japan, Vol. 41, No. 1, 203-212,1991

2. On reduction properties, J. Symbolic Logic, Vol. 59, No. 3, 900-911, 1994 (with A. Tsuboi)

\section{Kobayashi, Kojiro}

1. $\sum_{n}^{0}$ complete properties of programs and Martin-Löf randomness, Infor mation Processing Letters, vol, 46, 37-42, 1993

2. On malign input distributions for algorithms, IElCE Trans. on Information and Systems, vol. E76-D, no. 6, 634-640, 1993

3. Transformations that preserve malignness of universal distributions, Proceedings of First Annual International Conference, COCOON '95, Xi'an, China, 24-27, Aug. 1995, "Computing and Combinatorics" (Ed. by DingZhu Du and Ming Li), Lecture Notes in Computer Science, vol. 959, 420429 springer-Verlag, 1995

\section{Kudoh, $\mathbf{M}$}

1. On Some Oracle Results on the Bounded Error Probabilistic Polynomial Time Complexity Class BPP, in Proceedings of Logic Colloquium '95, Haifa, Israel, 1995 (with H. Tanaka)

\section{Kumabe, Masahiro}

1. A 1-generic degree which bounds a minimal degree, Journal of Symbolic Logic, vol 55, pp 733-743, 1990

2. Relative Recursive Enummerability of Generic degrees, Journal of Symbolic Logic, vol 56, pp 1075-1085, 1991

3. Every $n$-generic degree is a minimal cover of an n-neneric degree, Joural of symbolic Logic, vol 58, pp 219-231, 1993

4. Generic degrees are complemented, Annals of Pure and Applied Logic, vol 59, pp 257-272, 1993

5. Minimal upper bounds for the Arighmetical degrees, Journal of Sumbolic 
Logic, vol 59, pp 516-528, 1994

6. Degrees of generic sets, Computability, Enumerability, Unsolvability, Directions in recursion theory, Cambridge University Press, Lecture note serives, vol. 224, pp 1670184, 1996

\section{Matsubara, Yo}

1. Saturated ideals and the singular cardinal hypothesis, The Journal of Symbolic Logic, 57, 970-974, 1992

2. Ideals and combinatorial principles (with D. Burke), to appear in The Journal of Symbolic Logic

3. Nowhere precipitousness of some ideals, to appear in The Journal of Symbolic Logic (with M. Shioya)

4. Saturation problem of nonstationary ideal on $P_{k} \lambda$ (with D. Burke) (tentative title)

\section{Miyamoto, Tadatoshi}

1. $\omega_{1}$-Souslin trees under countable support iterations, Fundamenta Mathematicae, vol. 142, pp. 257-261, 1993

\section{Mori, Takakazu}

1. A metatheory of nonstandard analysis. Tsukuba J. Math., 17-1, 251-265, 1993 (with Tsujii, M, Yasugi) Mochizuki, Asae

1. A note on the functions which are not polynomial time computable from their graphs, Annals of the Japan Association for Philosophy of Science, Vol. 9, 17-22 (with J. Shinoda), 1996

\section{Muraki, Hisato}

1. Non-complementedness and non-distributivity of Kleene degrees, Mathmatical Logic Quarterl, to appear

\section{Murakami, M}

1. A nonstandard proof of the continuity of zeros of Polynomials, J. Fac. Sci. Univ. Tokyo Sect. IA, Math. 40 (1993), 53-54.

\section{Nagamachi, Shigeaki}

1. Nonstandard Analysis of Linear Canonical Transformations on a Fermion Fock Space with an Indefinite Metric. Osaka J. Math. 28, 579-607, 1991 (withT. Nishimura) 


\section{Nagashima, T}

1. See Kaneko [1]

\section{Nakamura, Toru}

1. A nonstandard representation of Feynman's path integrals. J. Math. Physics, 32-2, 457-463,1991

\section{Nakata, Masahiro}

1. NDK and Natural Reasoning, Manuscript, 1996 (with M. Yasugi) Nishimura, Takeshi See Nagamachi [1]

\section{Ogiwara, Masami}

1. On polynomial time bounded truth-table reducibility of NP sets to sparse sets, SlAM J. on Computing, 20, 471-483, 1991 (with O. Watanabe)

See also Toda [2

\section{Ojima, I.}

1. Unitary Representation of the Hyperfinite Heisenberg Group and the Logical Extension Methods in Physics, Open Systems and Information, Dynamics, 2 (1993), 107-128. (with M. Ozawa)

\section{Ozawa, Masanao}

1. Scott imcomplete Boolean ultrapowers of the real line. J. Symbolic Logic 60-1, 160-171, 1995

2. Forcing in nonstandard analysis. Ann. Pure Appl. Logic, 68-3, 263-297, 1994

See also Ojima [1], Hinokuma [1]

\section{Ryu, Katsuaki}

1. NDK, A New Classicall System, The Bull. ICS, Kyoto Sangyo University vol. II, 1-25, 1994 (with M. Yasugi)

\section{Shinoda, Juichi}

1. Strong polynomial time reducibility, Annals of Pure and Applied Logic, to appear See AoKi[1], [2] and Mochizuki [1]

\section{Shioya, Masahiro}

1. Weakly normal closures of filters on $P_{k} \lambda$, Jour. Symbolic Logic 58, 55-63, 1993

2. Infinitary Jónsson functions and elementary embeddings, Arch. Math. 
Logic 3381-86, 1994

3. The minimal normal $\mu$-complete filter on $P_{k} \lambda$, Proc. Amer. Math. Soc. 123, 1565-1572, 1995

See also Matsubara [3]

\section{Shirahata, Masaru}

1. A linear conservative extension of Zermelo-Fraenkel set theory, Studia Logica 56, 361-392, 1996.

\section{Tanaka, Hisao}

1. Some Descriptive-Set-Theoritical Problems in Complexity Theory, Publ. RlMS Kyoto Univ., Vol. 28, 603-614, 1992

2. Determining the Levels of Some Special Complexity Classes of Sets in the Kleene Arithmetical Hierarchy, Tokyo Journal of Math., Vol. 17, 125-133, 1994

See also Kudoh [1]

\section{Tanaka, Katusmi}

1. A two decomposition of a bounded metric space, Applied Mathematics Letters, Vol. 4, No. 1, 21-23, 1991 (joint work)

\section{Tanaka, Kazuyuki}

1. Weak axioms of determinacy and subsystems of analysis II, Ann. Pure and Applied Logic 52, 181-193, 1991

2. A note on the proof method of constructive falsity, Zeitschrift fur Math. Logik und Grund. Math. 37, 63-64, 1991

3. A game-theoretic proof of analytic Ramsey theorem, Zeitschrift fur Math. Logik und Grund. Math. 38, 301-304, 1992

4. Reverse Mathematics and subsystems of second-order arithmetic, American Math. Soc., Sugaku Expositions 5, 213-234, 1992

5. The self-embedding theorem of $W K L_{0}$ and a non-standard method, to appear in Annals of Pure and Applied Logic, 1996

6. Non-standard analysis in $W K L_{0}$, to appear in Math.Logic Quaterly

See also Kikuchi [2]

\section{Takahashi, Joji}

1. The stationary preserving part of a complete Boolean algebra, Kobe Jour nal of Mathematics, Vol. 10, 189-198, 1993 
Takahashi, Makoto

1. On $L_{[x}$-free Boolean algebras, Annals of Pure and applied Logic Vol. 55, 265-284, 1992 (with S. Fuchino and S. Koppelberg)

\section{Takaki, Osamu}

1. Normalization of Natural Deductions of a Constructive Arithmetic with Transfinite, Recursion and Bar Induction, Submitted, 1996

\section{Takeuti, Gaisi}

1. Bounded Arithmetic and the Polynomial Hierarchy, Annals of Pure and Applied Logic 52, 143-153, 1991 (with J. Krajícek and P. Pudlák)

2. A second order version of $S_{2}$ and $U_{2}^{1}$, J. Symbolic Logic 56, 1038-1063, 1991

3. Gaisi Takeuti, On Induction-Free Provability, Annals of Mathematics and Artificial Intelligence 6, 107-126, 1992 (with J. Krajicek)

4. Bounded arithmetic for NC, AlogTIME, NLSPACE and LSPACE, Annals of Pure and Applied Logic 56, 73-117, 1992 (with P. Clote)

5. Gaisi Takeuti, RSUV Isomorphisms, Proof Theory and Computational Complexity, P. Clote and J. Krajícek, eds., Oxford Univ. Press, 364-386, 1993

6. Provably Total Functions in Bounded Arithmetic Theroies $R_{3}^{i}, U_{2}^{i}$ and $V_{2}^{i}$, Proof Theory and Computational Complexity, P. Clote and J. Krajicek, eds., Oxford Univ. Press, 116-161, 1993 (with S.R. Buss and J. Krajícek)

7. The critical number of variables in a function, J. Symbolic Logic, 59, 12741284,1994

8. First order Bounded Arithmetic and small boolean circuit complexity classes, Feasible Mathematics Il, edited by P. Clote and J.B. Remmel, 153218, Birkhauser, 1995

9. Grzegorzgk hierarchy and Iep $\Sigma_{1}$, J. Symbolic Logic, 59, 1228-1244, 1994

10. RSUV Isomorphisms for $T A C^{i}, T N C^{i}$ and $T L S$, Archives for Mathematical Logic, 33, 427-453, 1995

11. Separations of Theories in Weak Bounded Arithmetic, Annals of Pure and Applied Logic, 71, 47-67, 1995

12. Forcing on Bounded Arithmetic, Gödel '96 edited by P. Hájek, 120-138, Lecture Notes in Logic, Springer, 1996 (with M. Yasumoto)

Toda,

1. Counting classes are at least as hard as the polynomial hierarchy, SlAM J. on Computing, 21, 316-328, 1992 (with M. Ogiwara)

2. $\mathrm{PP}$ is as hard as the polynomial-time hierarchy, SlAM J. on Computing, 20, 865-877, 1991 


\section{Tsujii, Yoshiki}

See Mori [1]

\section{Tsuboi, Akito}

1. Reduction peoperty and dimensional order property, in preparation

2. On definability of normal subgroup of superstable group, Zeitschrift fur Mathematische Logik und Grundlagen der Mathematik, vol 38, issue 2, 101106,1992

3. Algebraic types and automorphism groups, The Journal of Symbolic Logic, vol 58, No 3, 232-239, 1993

4. Large indiscernible sets of a structure, Kobe Journal of Mathematics, vol 10, No 2, 173-178, 1993

5. Amalgamations preserving $\boldsymbol{\aleph}_{0}$-categoricity, to appear in the JSL, December, 1996 (with A. Pillay)

See also Ikeda [3], [5], Kikyo [2]

\section{Tsuda, Teruko}

See AoKi [1], [2]

\section{Wakai, Kentaro}

1. Reduction peoperty and dimensional order property, to appeqr in Tsukuba Journal of Mathematics

\section{Wolter, F.}

1. Advanced Modal Logic, to appear in the second edition of Handbook of Philosophical Logic (with M. Zakharyaschev and A. Chagrov)

\section{Watanabe,}

See Ogiwara [1]

\section{Yasugi, Mariko}

See Hayashi [1], Hayahsi [2], Mori [1], Nakata [1] and Ryu [1].

\section{Yasumoto, Msahiro}

1. Nonstandard arithmetic and Hilbert subsets:Annals of Pure and Applied logic, 52, 195-202, 1991

2. Arithmetically Independent Integers and Values of Rational Functions Manuscripta Mathematica, 85, 1-10, 1994

See also Takeuti [12]

\section{Yoshinobu, Yasuo}

1. On precipitous ideals over the successor of limit cardinals, Master Thesis, 
Nagoya University, 1994

2. On Strength of Precipitousness of Some Ideals and Towers, submitted

\section{Yuasa, Yoshihumi}

1. Entangled Linear Orders in the Easton's Models, Tokyo Journal in Math ematics, Vol. 16, No. 2, pp 363-370, 1993

2. Adding a Cohen Real Adds an Entangled Linear Order, Archive for Math ematical Logic, Vol. 32, No. 4, 299-394, 1993

See also Eda [4] and Kada [1].

\section{Zakharyaschev, M}

See Wolter [1] 